\title{
High-resolution structures of immature and chimeric flaviviruses reveal key features redefining viral architecture and maturation
}

\author{
Joshua Hardy ${ }^{1, *}$, Natalee Newton ${ }^{2, *}$, Naphak Modhiran ${ }^{2}$, Connor Scott ${ }^{2}$, Hari Venugopal ${ }^{1}$, Laura Vet ${ }^{2}$, Paul Young ${ }^{2}$, \\ Roy Hall ${ }^{2}$, Jody Hobson-Peters ${ }^{2}$, Daniel Watterson ${ }^{2, *}$, Fasseli Coulibaly ${ }^{1, *}$ \\ ${ }^{1}$ Monash University, Clayton, VIC, Australia; \\ ${ }^{2}$ The University of Queensland, Brisbane, QLD, Australia; *authors contributed equally.
}

fasseli.coulibaly@monash.edu; d.watterson@uq.edu.au

Flaviviruses pose a complex threat to human health including a few global pathogens and numerous viruses with an epidemic potential. In the context of the co-circulation of closely-related viruses, non-neutralising immune responses may aggravate subsequent heterologous infections. Sub-optimal responses to vaccination entails a similar risk. To address these challenges, a detailed structural understanding of flavivirus infectious particles is essential to characterise quaternary epitopes responsible for broadly protective responses or, on the contrary, deleterious immune responses. Immature-like features and conformational "breathing" in circulating virions have been linked to the latter prompting for a better understanding of structural transitions underpinning viral maturation.

Taking advantage of an insect-specific flavivirus (ISF), we have determined high-resolution structures of immature and mature particles revealing key features in the maturation process. First, we produced chimeric viruses between the ISF and medically-relevant flaviviruses. We show that the outer shell of the chimeric viruses is native, which allowed cryo-EM structure determination at highresolution for West Nile virus, Murray Valley Encephalitis virus and dengue virus. The structure of the dengue virus chimera at a resolution of $2.5 \AA$ reveals lipid-like ligands with a structural role likely to be conserved across all pathogenic flaviviruses. The structure of the immature ISF particle at a resolution of 3.9 $\AA$ shows how the stem region of the E protein, where these ligands bind, is remodelled during maturation. Unexpectedly, the immature spike adopts a topology where prM forms a central pillar rather than the peripheral drawstring proposed earlier (Fig. 1A). This topology implies a revised organisation of the immature virion, which supports a collapse model for viral maturation (Fig. 1B). In this model, folding down of prM onto the membrane guides the collapse of the trimeric spikes.

Together, these structures provide new avenues to target the stem regions of $\mathrm{E}$ and prM for the development of improved vaccines and new therapeutics. More generally, we propose that the chimeric platform could be a largely applicable tool to investigate flavivirus biology.

A

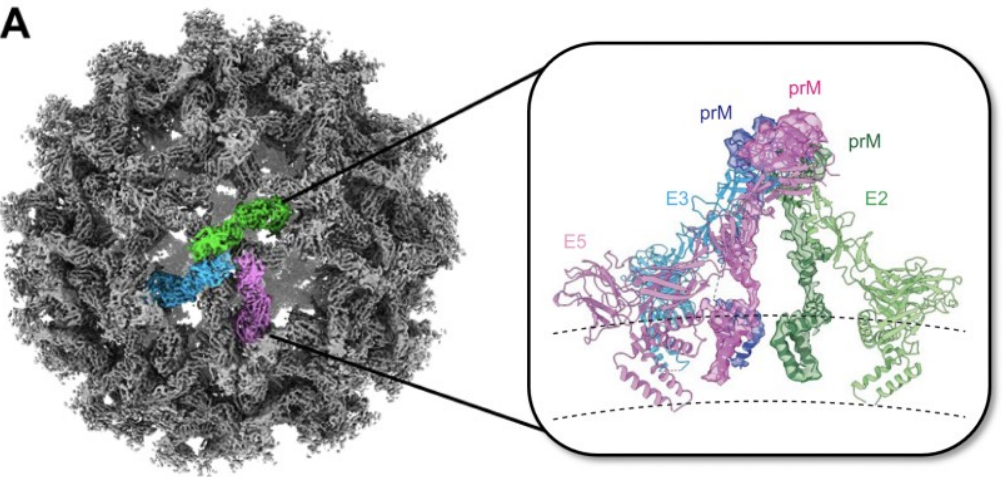

B

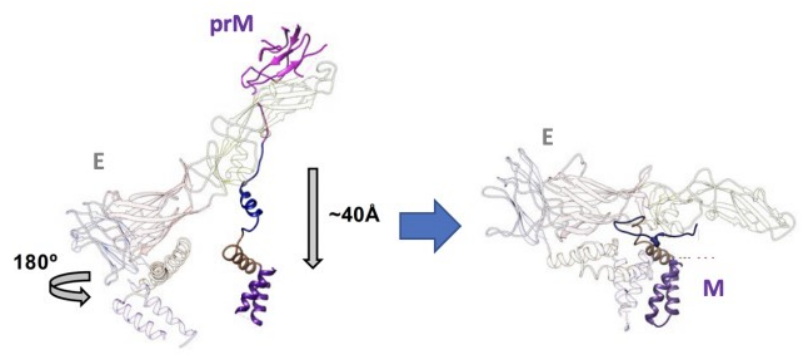

Figure 1. (A) Structure of the immature Binjari virus. The inset represents a spike, with E subunits forming a splayed tripod buttressed by a central pillar of prM subunits. (B) Comparison of the heterodimers (pr)M-E in the immature and mature particles.

Keywords: flaviviruses; virus assembly; cryo-EM; epidemic; insect viruses 